\title{
Strain and localization effects in InGaAs(N) quantum wells: Tuning the magnetic response
}

\author{
V. Lopes-Oliveira, ${ }^{1, a)}$ L. K. S. Herval, ${ }^{1}$ V. Orsi Gordo, ${ }^{1}$ D. F. Cesar, ${ }^{1}$ M. P. F. de Godoy, ${ }^{1}$ \\ Y. Galvão Gobato, ${ }^{1}$ M. Henini, ${ }^{2}$ A. Khatab, ${ }^{2,3}$ M. Sadeghi, ${ }^{4}$ S. Wang, ${ }^{4}$ and M. Schmidbauer ${ }^{5}$ \\ ${ }^{1}$ Departamento de Física, Universidade Federal de São Carlos, 13565-905, São Carlos, São Paulo, Brazil \\ ${ }^{2}$ School of Physics and Astronomy, Nottingham Nanotechnology and Nanoscience Centre, University of \\ Nottingham, NG7 2RD Nottingham, United Kingdom \\ ${ }^{3}$ National Institute of Laser Enhanced Sciences, Cairo University, Egypt \\ ${ }^{4}$ Photonics Laboratory, Department of Microtechnology and Nanoscience, Chalmers University of \\ Technology, 41296 Goteborg, Sweden \\ ${ }^{5}$ Leibniz-Institute for Crystal Growth, Max-Born-Str. 2, D-12489 Berlin, Germany
}

(Received 26 June 2014; accepted 4 December 2014; published online 18 December 2014)

\begin{abstract}
We investigated effects of localization and strain on the optical and magneto-optical properties of diluted nitrogen III-V quantum wells theoretically and experimentally. High-resolution x-ray diffraction, photoluminescence (PL), and magneto-PL measurements under high magnetic fields up to $15 \mathrm{~T}$ were performed at low temperatures. Bir-Pikus Hamiltonian formalism was used to study the influence of strain, confinement, and localization effects. The circularly polarized magneto-PL was interpreted considering localization aspects in the valence band ground state. An anomalous behavior of the electron-hole pair magnetic shift was observed at low magnetic fields, ascribed to the increase in the exciton reduced mass due to the negative effective mass of the valence band ground state. (C) 2014 AIP Publishing LLC. [http://dx.doi.org/10.1063/1.4904357]
\end{abstract}

\section{INTRODUCTION}

Electronic structure of semiconductor systems is sensitive to alloy compositions, confinement, strain, and magnetic fields. The simultaneous combination of such effects results in particular, behaviors. For instance, introducing small amounts of nitrogen $(\mathrm{N})$ in conventional III-V arsenidebased alloys causes a drastic decrease in the bandgap emission due to the coupling between the bottom conduction band and the top of the resonant band of localized nitrogen. ${ }^{1,2}$ This fact has drawn much attention due to their potential applications in optoelectronic devices in the optical range of $1.30-1.55 \mu \mathrm{m}$, which is interesting for telecommunications. However, the presence of $\mathrm{N}$ usually introduces strain, as well as alloy imperfections (localized states) which significantly alter the optical emission in different ways. ${ }^{3-6}$ In fact, using nitrogen in $\mathrm{InGaAsN}$ results in an undesirable deterioration of its optical quality. It is widely known that the broad photoluminescence (PL) band, which is attributed to the recombination of localized excitons (LEs), consists of narrow lines related to the recombination of individual localized excitons. ${ }^{7,8}$ These localization effects in N-diluted III-V semiconductors are attributed to electrons trapped in alloy imperfections which were introduced during the growth at a low temperature. ${ }^{8-12}$ A discussion on the enhancement of electron effective mass due to the conduction band interaction and the nitrogen alloy imperfection state above the gap has been studied in the literature. ${ }^{13,14}$ This leads to a peculiar reduction in the diamagnetic shift detected in the optical recombination of excitons. Yet, as also reported in Ref. 13, unusually large in-plane masses can also be found, and as

a)lopes@df.ufscar.br described here, even negative diamagnetic shifts could be expected. These additional effects can be ascribed to exciton localization in alloy imperfections, as will be shown in this article.

The focus of this study is the correlation between spinresolved optical emission, strain, and confinement modulation produced by defects in nitrogenated heterostructures. Within the framework of a multiband calculation, these effects can be combined with strain. The biaxial strain produced at the interfaces is introduced into the Pikus-Bir Hamiltonian. ${ }^{15}$ The hydrostatic strain component renormalizes the gap $\left(\delta E_{h}\right)$ proportionally to the deformation potential $a_{d}=a_{v}+a_{c}$, with $a_{v}$ the valence band contribution and $a_{c}$ the contribution of the conduction band. In turn, the shear strain leads to the relative shift $\left(\delta E_{s}\right)$ between the top valence subbands, heavy $E_{h h}$, and light-hole, $E_{l h}$. Therefore, the impact of confinement, strain, and alloy imperfection effects are discussed based on our experimental results in optical properties of [100] double InGaAsN/GaAs and InGaAs/GaAs (reference) quantum wells (QWs).

The paper is organized as follows: Section II describes the experimental methods, Sec. III explains the details of the electronic structure calculation, and Sec. IV discusses the main results. The concluding remarks are presented in Sec. V.

\section{EXPERIMENT}

Our samples were grown by Molecular Beam Epitaxy (MBE) using a Varian Gen-II system on a semi-insulating (100)GaAs substrate. The samples consist of a double QW structure as follows: $100 \mathrm{~nm}$ GaAs buffer layer, $100 \mathrm{~nm}$ $\mathrm{Al}_{0.35} \mathrm{Ga}_{0.65} \mathrm{As}$ barrier, $100 \mathrm{~nm}$ GaAs barrier, $7 \mathrm{~nm}$ InGaAsN 
QW, $100 \mathrm{~nm}$ GaAs barrier, $4 \mathrm{~nm}$ InGaAsN QW, $100 \mathrm{~nm}$ GaAs barrier, $100 \mathrm{~nm} \mathrm{Al}_{0.35} \mathrm{Ga}_{0.65} \mathrm{As}$ barrier, and $10 \mathrm{~nm}$ GaAs cap layer. The nominal concentrations of In and $\mathrm{N}$ are $36 \%$ and $1.2 \%$, respectively. The growth temperature was $600{ }^{\circ} \mathrm{C}$ except for the QWs which were grown at $450{ }^{\circ} \mathrm{C}$. The QWs are labeled QWN4 and QWN7 for $4 \mathrm{~nm}$ QW width and $7 \mathrm{~nm}$ QW width, respectively. The reference sample was grown by using the same parameters except for the QWs which consist of $\operatorname{In}_{0.36} \mathrm{Ga}_{0.64} \mathrm{As}$ QWs. ${ }^{16}$ All samples were characterized by high-resolution $\mathrm{x}$-ray diffraction (HRXRD) employing the $C u-K_{\alpha 1}$ line $(\lambda=1.54056 \AA)$ for symmetrical (400) reflections in (100)-substrates. PL measurements were performed in the range of $10-300 \mathrm{~K}$ in a Janis cryostat. Circularly polarized magneto-photoluminescence was investigated using appropriate optical components in a Faraday configuration at $4 \mathrm{~K}$ under magnetic fields up to $15 \mathrm{~T}$. All samples were excited by a $532 \mathrm{~nm}$ diode laser at an intensity of $10^{2} \mathrm{~W} \mathrm{~cm}^{-2}$ and the PL signal collected in a micro-PL setup by a $0.5 \mathrm{~m}$ Andor spectrometer coupled with an InGaAs array diode.

\section{ELECTRONIC STRUCTURE}

As the samples were grown along the direction [100], the representation of the Hamiltonian in the presence of a magnetic field is given by a $4 \times 4$ matrix

$$
H_{L}^{i j}=H_{K}^{i j}+V(r) \delta_{i j},
$$

where $H_{K}$ is the Luttinger Hamiltonian in the axial approximation, ${ }^{17,18}$ which includes the magnetic field applied along the direction $z$ within the same framework. The potential profile $V(r)$ for the heavy and light-holes is introduced to the diagonal terms of $H_{K}$. The Hamiltonian $H_{K}$ is given by

$$
H_{K}=\left(\begin{array}{cccc}
a_{+}^{h h \uparrow} & b_{-} & c_{-} & 0 \\
b_{+} & a_{-}^{l h \uparrow} & 0 & c_{-} \\
c_{+} & 0 & d_{-}^{l h \downarrow} & b_{-} \\
0 & c_{+} & b_{+} & d_{+}^{h h \downarrow}
\end{array}\right) .
$$

The matrix elements are

$$
\begin{gathered}
a_{ \pm}=-\frac{\hbar^{2}}{2 m_{0}}\left(\gamma_{1} \mp 2 \gamma_{2}\right) k_{z}^{2}-\frac{\hbar^{2}}{4 m_{0}}\left(\gamma_{1} \pm \gamma_{2}\right)\left(k_{+} k_{-}+k_{-} k_{+}\right) \\
+\frac{(2 \pm 1)}{2} \hbar \omega_{e}\left(\kappa+\frac{(5 \pm 4)}{4} q\right), \\
d_{ \pm}=a_{ \pm}-\frac{(2 \pm 1)}{2} \hbar \omega_{e}\left(\kappa+\frac{(5 \pm 4)}{4} q\right), \\
b_{\mp}=\hbar^{2} \frac{\sqrt{3}}{4 m_{0}} \gamma_{3} k_{z} k_{\mp}, \\
c_{\mp}=\hbar^{2} \frac{\sqrt{3}}{4 m_{0}}\left(\gamma_{2}+\gamma_{3}\right) k_{\mp}^{2}
\end{gathered}
$$

where

$$
k_{ \pm}=-i e^{ \pm i \varphi}\left(\frac{\partial}{\partial r} \pm \frac{i}{r} \frac{\partial}{\partial \varphi} \mp \frac{m_{0} \omega_{e}}{2 \hbar \lambda^{2}} r\right), \quad k_{z}=-i \frac{\partial}{\partial z},
$$

$m_{0}$ is the electron mass, $\lambda$ is the effective magnetic length of the QW, the cyclotron frequency is given by $\omega_{e}=\frac{e B}{m_{0} c}$, and $q$, $\kappa, \gamma_{\alpha}(\alpha=1,2,3)$ are the Luttinger parameters. Parameters $q$ and $\kappa$ give the Zeeman contribution and $\gamma_{\alpha}$ can be obtained from the anisotropic effective masses or calculated from the first principles.

The split-off and valence bands are separated in more than $300 \mathrm{meV}$. Therefore, we have neglected split-off effects and used a Luttinger Hamiltonian with strain field because the valence sub-bands effects are much more significant than split-off bands effects. The contribution of strain to the valence sub-bands $h h$ and $l h$ is given by ${ }^{19}$

$$
\begin{aligned}
& \delta E_{h h}=-(P+Q)=-\frac{1}{2}\left[\beta_{1}\left(2 \varepsilon_{\|}+\varepsilon_{\perp}\right)\right]-\beta_{2} \varepsilon_{\| \perp} \\
& \delta E_{l h}=-(P-Q)=-\frac{1}{2}\left[\beta_{1}\left(2 \varepsilon_{\|}+\varepsilon_{\perp}\right)\right]+\beta_{2} \varepsilon_{\| \perp}
\end{aligned}
$$

with $\quad \varepsilon_{\| \perp}=\varepsilon_{\|}-\varepsilon_{\perp}, \quad \varepsilon_{\perp}=-2 \frac{C_{12}}{C_{11}} \varepsilon_{\|}, \quad \varepsilon_{\|}=\frac{a_{S}-a_{L}}{a_{L}}$, where $\beta_{1}=-2 a_{v}, \beta_{2}=b, a_{S}$ is the lattice parameter of the substrate (GaAs) and $a_{L}$, for the material in the quantum well; $a_{c}, a_{v}$, $b$, and $d$ are the deformation potentials, and $C_{i j}$ are the elastic constants. According to the sign of the strain in the quantum well, the relative shift of the $h h$ and $l h$ sub-band may drastically affect the electronic structure. By changing their relative position, the effective coupling between them will certainly be altered. In Fig. 1, we show how compressive or tensile strain affects the relative positions of the bottom of the conduction band and the tops of the $h h$ and $l h$ sub-bands. A compressive strain $\left(\varepsilon_{\|}<0\right)$ increases the emission energy for both an optical recombination involving electron- $l h$ and electron- $h h$. Yet, the electron- $h h$ transition shift is less significant as the $h h$ and conduction band move in the same direction.

Additionally, it has been discussed at length ${ }^{20,21}$ that, given the low temperature at which the InGaAsN QWs are grown, the appearance of interface alloy imperfections due

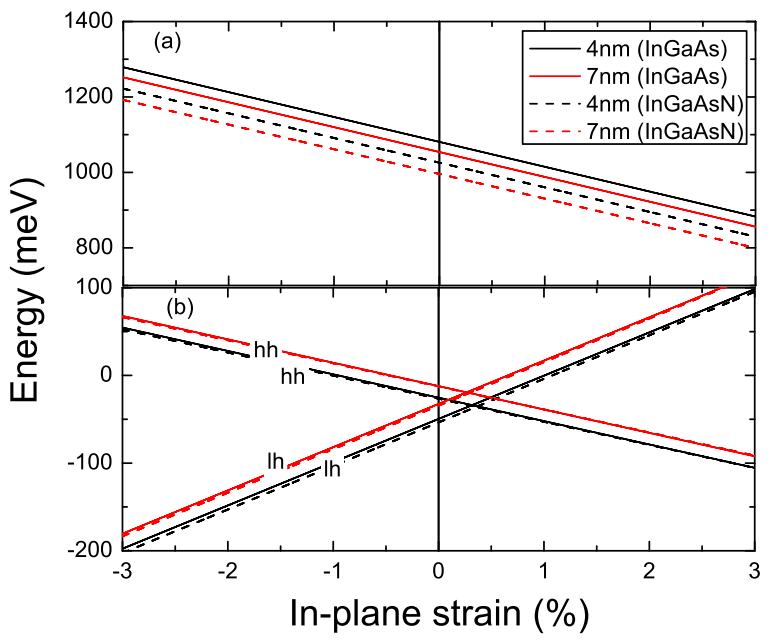

FIG. 1. (a) Comparison between the conduction band energies as a function of strain for the profiles corresponding to the samples of the InGaAs and InGaAsN quantum well for two different sizes: 4 and $7 \mathrm{~nm}$. (b) Comparison between the valence subbands energies as a function of strain for the profiles corresponding to the samples of the InGaAs and InGaAsN quantum well for two different sizes: 4 and $7 \mathrm{~nm}$. 
TABLE I. Calculated parameters for the samples $\operatorname{In}_{0.36} \mathrm{Ga}_{0.64} \mathrm{As}$ e $\operatorname{In}_{0.36} \mathrm{Ga}_{0.64} \mathrm{As}_{0.088} \mathrm{~N}_{0.012}{ }^{32}$

\begin{tabular}{|c|c|c|c|c|c|c|}
\hline Parameters & $\mathrm{GaN}$ & $\mathrm{GaAs}$ & InAs & $\mathrm{InN}$ & $\mathrm{In}_{0.36} \mathrm{Ga}_{0.64} \mathrm{As}$ & $\mathrm{In}_{0.36} \mathrm{Ga}_{0.64} \mathrm{As}_{0.988} \mathrm{~N}_{0.012}$ \\
\hline$E_{g}(\mathrm{eV})$ & 3.299 & 1.519 & 0.417 & 1.94 & 1.0106 & 0.9503 \\
\hline$a_{c}(\mathrm{eV})$ & -2.2 & -7.17 & -5.08 & -1.85 & -6.417 & -6.365 \\
\hline$a_{v}(\mathrm{eV})$ & -0.69 & -1.16 & -1.00 & -0.70 & -1.102 & -1.097 \\
\hline$b(\mathrm{eV})$ & -2.20 & -2.00 & -1.80 & -1.20 & -1.928 & -1.927 \\
\hline$d(\mathrm{eV})$ & -3.4 & -4.8 & -3.6 & -9.3 & -4.368 & -4.382 \\
\hline$C_{11}(\mathrm{GPa})$ & 293 & 1221 & 833 & 187 & 1081 & 1071 \\
\hline$C_{12}(\mathrm{GPa})$ & 159 & 566 & 453 & 125 & 525 & 521 \\
\hline$C_{44}(\mathrm{GPa})$ & 155 & 600 & 396 & 86 & 526 & 522 \\
\hline$a(\mathrm{~nm})$ & 0.452 & 0.565 & 0.606 & 0.498 & 0.580 & 0.578 \\
\hline$m^{*}$ & 0.15 & 0.067 & 0.026 & 0.12 & 0.052 & 0.053 \\
\hline$\gamma_{1}$ & 2.67 & 6.98 & 20.0 & 3.72 & 11.67 & 11.56 \\
\hline$\gamma_{2}$ & 0.75 & 2.06 & 8.5 & 1.26 & 4.38 & 4.34 \\
\hline$\gamma_{3}$ & 1.10 & 2.93 & 9.2 & 1.63 & 5.19 & 5.14 \\
\hline$g$ & $\ldots$ & -0.44 & -15 & $\ldots$ & -5.68 & $\ldots$ \\
\hline
\end{tabular}

to the changing of the band gap originating from the $\mathrm{N}$ addition, is practically unavoidable. Thus, some of the parameters needed to be determined to build the k.p Hamiltonian that are not currently reported. Thereby, interpolation procedures should be put in place. For both the ternary $\mathrm{A}_{x} \mathrm{~B}_{1-x} \mathrm{C}$ and quaternary $\mathrm{A}_{x} \mathrm{~B}_{1-x} \mathrm{C}_{1-y} \mathrm{D}_{y}$ alloys, we interpolated between reported values of band parameters corresponding to the relevant binary alloys. The relevant parameters obtained by interpolation using the expressions in Ref. 22 are listed in Table I. However, for the energy gap, the nitrogen content causes an anomalous shrinking impossible to emulate by simple interpolation.

Note that for both $\mathrm{GaN}$ and $\mathrm{InN}$, the value of the energy gap exceeds the one for InGaAs regardless of the In content. Thus, we used available alternative models to obtain the gaps of the ternary $E_{g}^{I n_{x} G a_{1-x} A s}$ and quaternary alloys $E_{g}^{I n_{x} G a_{1-x} A s_{1-y} N_{y}}, 23-25$ which accounts for the coupling between the nitrogen resonant level and the conduction band edge. The band-offsets were calculated from the difference of the energy gaps between the wells and the substrates.

The qualitative effect of localization can be introduced into the discussion by assuming the presence of small localization cores of volume $\Omega$. The wave function $\Psi_{0}$, defined within $V_{0}$, transforms to $\Psi=\Psi_{0}+\phi$, where $\phi$ is defined within the volume $\Omega$, and the Hamiltonian, $H_{0}$, transforms to $H=H_{0}+\Delta H$, within the total volume $V_{T}=V_{0}+\Omega$. By defining, $\frac{1}{V_{0}} \int_{V_{0}} \Psi_{0}^{*} H_{0} \Psi_{0} d v=E_{0}, \frac{1}{\Omega} \int_{\Omega} \phi^{*} \Delta H \phi d v=\Delta E$, the eigenvalue of $H$ can be determined as $E=\frac{1}{V_{T}} \int_{V_{T}} \Psi^{*} H \Psi d v=$ $\frac{V_{0}}{V_{T}} E_{0}+\frac{\Omega}{V_{T}} \Delta E$. This can be approximated to $E=E_{0}+\frac{\Omega}{V_{T}} \Delta E$ in the case where $\Omega \ll V_{0}$.

For a cylindrical localization core, as represented in Fig. 2(a), according to Eq. (3), the diagonal energy values of the $h h$ and $l h$ valence band ground states localized within $\Omega$ are given by

$$
\Delta E_{h h(l h)}=-\frac{\hbar^{2} \pi^{2}}{2 m_{z}^{h h(l h)} \Delta_{z}^{2}}-\frac{\hbar^{2}\left(2 a_{1}\right)^{2}}{2 m_{x y}^{h h(l h)} \Delta_{x y}^{2}},
$$

where $m_{z}^{h h}=m_{0} /\left(\gamma_{1}-2 \gamma_{2}\right), m_{z}^{l h}=m_{0} /\left(\gamma_{1}+2 \gamma_{2}\right), m_{x y}^{h h}=m_{0} /$ $\left(\gamma_{1}+\gamma_{2}\right), m_{x y}^{l h}=m_{0} /\left(\gamma_{1}-\gamma_{2}\right), \Delta_{x y}$ and $\Delta_{z}$ are the effective dimensions of the localized state at the interface due to disorder and $a_{1} \simeq 2.4048$, is the zero of the Bessel function $J_{0}\left(a_{1}\right)=0$.

The corresponding correction to the energy difference between both valence sub-bands, $\delta E_{h h}-\delta E_{l h}=\frac{\Omega}{V_{T}}$ $\left(\Delta E_{h h}-\Delta E_{l h}\right)$, is given by
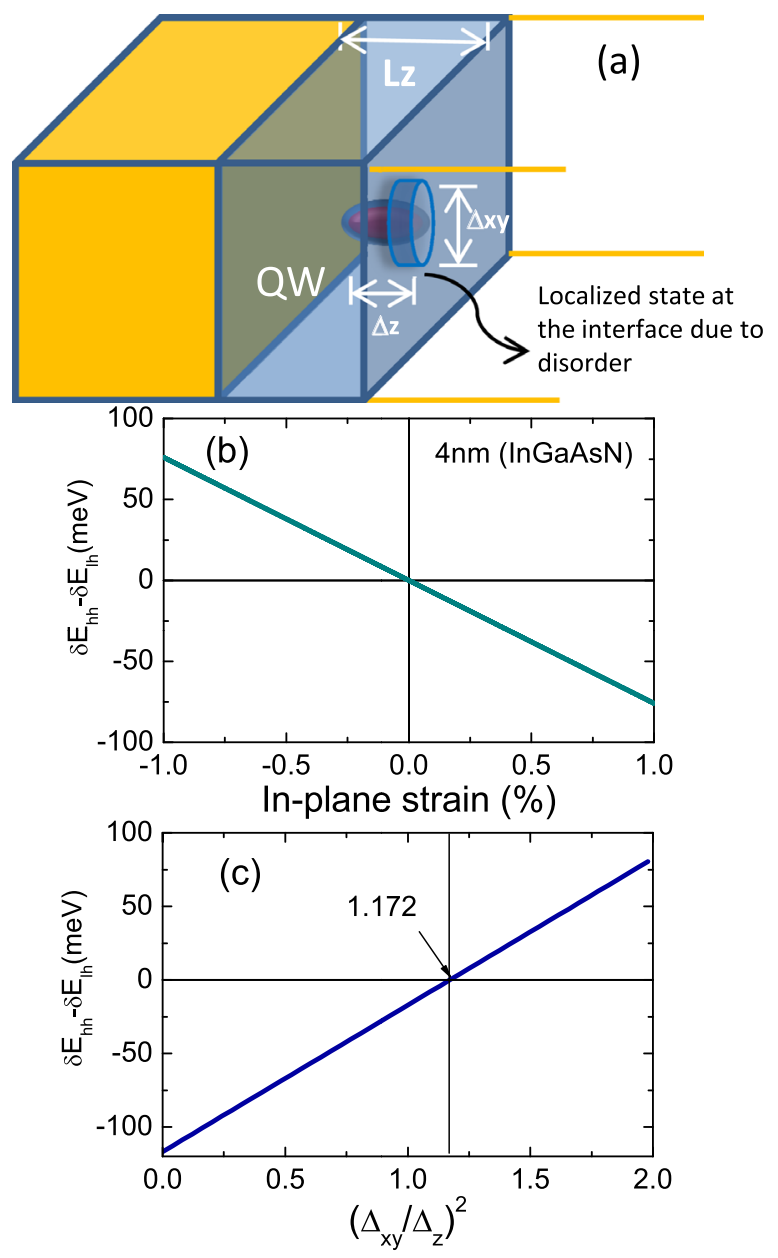

FIG. 2. Diagram representing the QW layer with a lateral localization core with cylindrical symmetry. (a) Relative valence sub-band shift, $\delta E_{h h}-\delta E_{l h}$, due to strain effects. (b) The same shift by considering only the localization effects as a function of the square aspect ratio. 


$$
\delta E_{h h}-\delta E_{l h}=\varepsilon_{0} \gamma_{2} \frac{\Delta_{z}}{L_{z}}\left[\pi^{2}\left(\frac{\Delta_{x y}}{\Delta_{z}}\right)^{2}-2 a_{1}^{2}\right],
$$

with $\varepsilon_{0}=\frac{\hbar^{2}}{2 m_{0} R_{e f f}^{2}}$, the binding energy for the unperturbed wavefunction confined within the QW and spread laterally within the effective Bohr radius $R_{\text {eff }}$.

To quantify the net effect of disorder within a single particle model, we may consider a local one monolayer fluctuation of the QW size, which according to Table I corresponds to $\Delta_{z} / L_{z} \sim 0.15$ for $L_{z}=4 \mathrm{~nm}$. Thus, we can quantitatively compare the relative subband shift, $\delta E_{h h}-\delta E_{l h}$, obtained from Eqs. (8) and (9) and shown in Fig. 2(b) as a function of strain, with the one from Eq. (11), as a function of the square aspect ratio, $\left(\frac{\Delta_{x y}}{\Delta_{z}}\right)^{2}$ by using $\varepsilon_{0}=17.5 \mathrm{meV}$ from Ref. 26 . The results shown in Figs. 2(b) and 2(c) illustrate that both strain modulation and the aspect ratio, in confined valence band states, tune the relative sub-bands position. This has a potential effect on the hybridization of valence sub-bands due to interband coupling, as described by the Hamiltonian in Eq. (2). This coupling modulates various effective parameters such as effective masses, Lande factors, and diamagnetic shifts, as well as their dependence on external fields. $^{27,28}$

\section{RESULTS}

The HRXRD results shown in Figure 3 show that the introduction of nitrogen induces a reduction in the lattice parameter along $\mathrm{z}$, leading to a shift of the diffraction angle toward the peak of GaAs.

For relaxed layers, the lattice parameter

$$
a(x, y)
$$

of a quaternary system (e.g., $\mathrm{In}_{x} \mathrm{Ga}_{1-x} \mathrm{As}_{1-y} \mathrm{~N}_{y}$ layer) can be calculated-e.g., by applying Vegard's law-when the atomic concentrations $\mathrm{x}$ and $\mathrm{y}$ are known. In our case, we can write for the (relaxed) lattice parameter of $\operatorname{In}_{x} \mathrm{Ga}_{1-x} \mathrm{As}_{1-y} \mathrm{~N}_{y}$ QWs

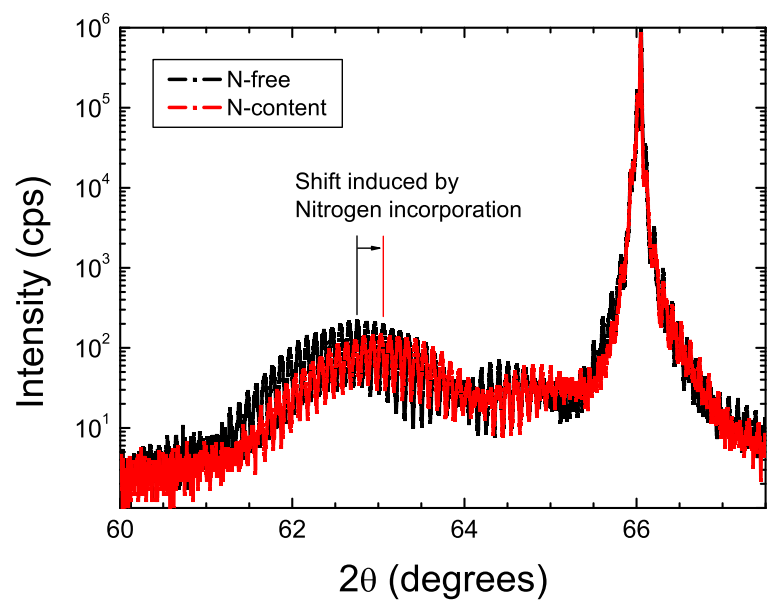

FIG. 3. Comparison between sample N-free (black line) and N-containing QW (red line) X-ray diffraction. The shift of the red broad peak to higher angles is induced by nitrogen incorporated into the two QWs.

$$
\begin{aligned}
a(x, y) & =a\left(\operatorname{In}_{x} \operatorname{Ga}_{1-x} \operatorname{As}_{1-y} N_{y}\right) \\
& =x \cdot a\left(\operatorname{InAs}_{1-y} N_{y}\right)+(1-x) a\left(\operatorname{GaAs}_{1-y} N_{y}\right) .
\end{aligned}
$$

From the first ${ }^{29}$ and second terms, ${ }^{30}$ we obtain that

$$
\begin{aligned}
a\left(\operatorname{In}_{x} \mathrm{Ga}_{1-x} \mathrm{As}_{1-y} N_{y}\right)= & a\left(\operatorname{In}_{x} \mathrm{Ga}_{1-x} \mathrm{As}\right)-y \cdot 1.0605 \AA \\
& -(1-x) \cdot y^{2} \cdot 20.95 \AA .
\end{aligned}
$$

This implies that the lattice parameter in the N-content QW is slightly lower than in the N-free QW. Therefore, the main influence of $\mathrm{N}$ in the structural properties of $\mathrm{QW}$ is the reduction in compressive strain due to a decreasing in the lattice parameter as compared to $\mathrm{N}$-free QW. This result is consistent with the behavior shown in Fig. 3, where the band related to N-content QW is near the substrate peak at (004) direction.

Figure 4 shows typical PL spectra for the reference and $\mathrm{N}$-containing double QWs at different temperatures. The PL spectra at $10 \mathrm{~K}$ reveal two emission peaks attributed to different QW widths: at $1.212 \mathrm{eV}$ and $1.153 \mathrm{eV}$ for the reference sample and at $1.112 \mathrm{eV}$ and $1.055 \mathrm{eV}$ for the $\mathrm{N}$ containing QWs. As expected, the PL peak energy presents a red shift for $\mathrm{N}$-containing QWs due to the reduction in energy gap with $\mathrm{N}$-content. We have particularly studied the temperature dependence of the PL peak energy and its PL intensity for both samples in the range 10-300 K. Figure 5 presents the theoretical and experimental results for the temperature dependence of the PL peak energy for both QWs with and without N. PL peak positions were estimated theoretically in the presence and absence of strain effects. In general, the decrease in emission energy as a function of temperature follows the Varshnii model ${ }^{31}$

$$
E_{g}(T)=E_{g}(0)-\frac{\alpha T^{2}}{T+\beta} .
$$

The values of the parameters used in the calculations are presented in Table I and were obtained by the interpolation procedure described above. Varshni's parameters were also

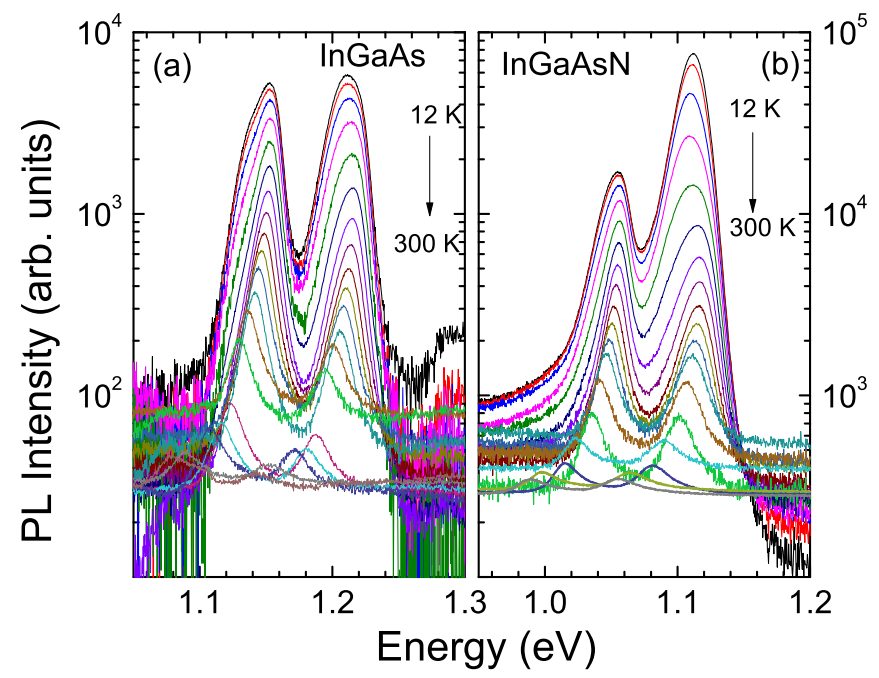

FIG. 4. PL spectra as a function of temperature (a) InGaAs (QW) reference sample and (b) InGaAsN (QWN) sample. 


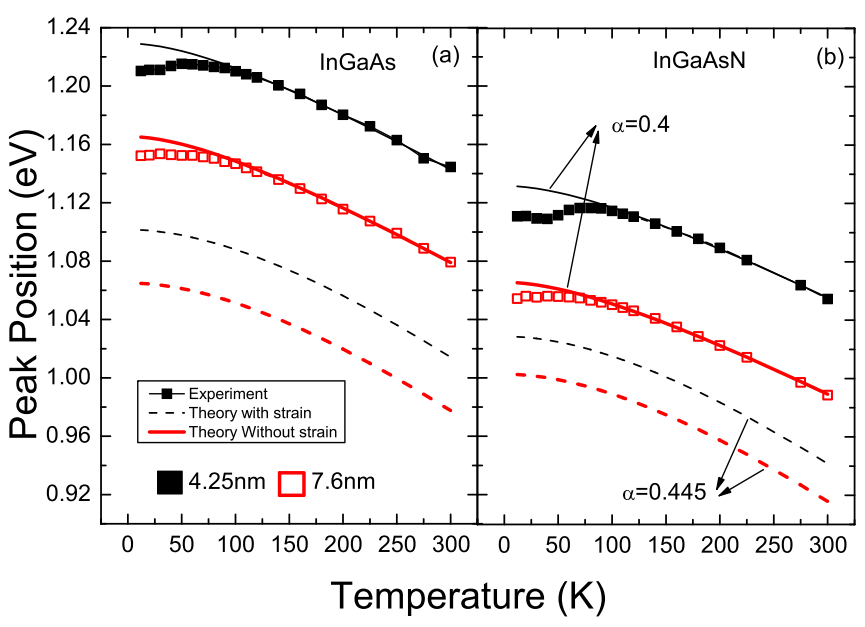

FIG. 5. Transition energies as a function of temperature. Experimental curves (lines and symbols) and calculated values (curves): with strain effects (solid curves) and without strain (dashed curves).

calculated by linear interpolation using the values obtained in Ref. 32 and are given in Table II.

If we ascribe the gap modification to strain effects, the values of the deformation $\varepsilon_{\|}$necessary to adjust the theoretical and experimental curves are given in Table III. It is worth noting that in the case of the $\mathrm{In}_{0.36} \mathrm{Ga}_{0.64} \mathrm{As} \mathrm{QWs}$, the values of Varshni's parameters obtained by the interpolation process yield an adequate adjustment with the experiment, yet for the nitrogenated alloy, the experiment could only be fitted by reducing the value of $\alpha$, as indicated in Fig. 5. In what follows, we will show that beside the strain effects, various features detected experimentally can be unambiguously ascribed to the confinement modulation.

It is well known that high density of structural defects is introduced during the low temperature growth of the QWs such as $450{ }^{\circ} \mathrm{C}$. We have observed a S-shape for the temperature dependence of the peak position which was associated to exciton localization by these imperfections. ${ }^{8,33}$ We remark that such S-shape behavior was observed for both samples and is mainly related to the low temperature growth conditions. As expected, the InGaAsN QWs show more significant S-shape behavior as compared to the reference sample. In fact, emissions at low temperatures are dominated by LEs trapped in deep states which results in lower energy emission. For higher temperatures, these emissions are dominated by delocalized excitons (DL) and the temperature dependence of PL peak energy follows the Varshnii expression. Therefore, the observed S-shape behavior cannot be explained by strain effects and is mainly associated to localization of carriers by structural defects.

Figure 6 presents the temperature dependence of PL intensity for both samples. The integrated PL intensity as a

TABLE II. Values of Varshni's parameters obtained by the interpolation $\left({ }^{\mathrm{a}} \alpha=0.4\right.$ was adjusted to the experimental curves).

\begin{tabular}{lcc}
\hline \hline & $\operatorname{In}_{0.36} \mathrm{Ga}_{0.64} \mathrm{As}$ & $\mathrm{In}_{0.36} \mathrm{Ga}_{0.64} \mathrm{As}_{0.988} \mathrm{~N}_{0.012}$ \\
\hline$E_{g}(0)(\mathrm{meV})$ & 1010.6 & 950.3 \\
$\alpha(\mathrm{meV} / \mathrm{K})$ & 0.445 & $0.445\left({ }^{\mathrm{a}} 0.4\right)$ \\
$\beta(\mathrm{K})$ & 164.04 & 169.37 \\
\hline \hline
\end{tabular}

TABLE III. Values of $\varepsilon_{\|}$used in Fig. 5.

\begin{tabular}{lcr}
\hline \hline Wells & Width & $\varepsilon_{\|}(\%)$ \\
\hline $\mathrm{In}_{0.36} \mathrm{Ga}_{0.64} \mathrm{As}$ & $4 \mathrm{~nm}$ & -2.62 \\
$\mathrm{In}_{0.36} \mathrm{Ga}_{0.64} \mathrm{As}$ & $7 \mathrm{~nm}$ & -2.14 \\
$\mathrm{In}_{0.36} \mathrm{Ga}_{0.64} \mathrm{As}_{0.988} \mathrm{~N}_{0.012}$ & $4 \mathrm{~nm}$ & -2.15 \\
$\mathrm{In}_{0.36} \mathrm{Ga}_{0.64} \mathrm{As}_{0.988} \mathrm{~N}_{0.012}$ & $7 \mathrm{~nm}$ & -1.24 \\
\hline \hline
\end{tabular}

function of temperature can be fitted by the phenomenological expression and to simplify the characteristic of integrated density as a function of temperature, we used two nonradiative processes: $\gamma(1)$ relative to localized and $\gamma(2)$ delocalized excitons, where the parameter (1) is related to the process predominant at low temperatures and (2) at high temperatures: ${ }^{34,35}$

$$
I(T)=\frac{I_{0}}{1+\gamma(1) e^{\frac{-E_{a}(1)}{k T}}+\gamma(2) e^{\frac{-E_{a}(2)}{k T}}},
$$

where $E_{a}(1,2)$ are the thermal activation energies, $\gamma(1,2)$ are the ratios between radiative and non-radiative lifetimes and $I_{0}$ is the intensity at $T=0 \mathrm{~K}$. The fitting parameters are presented in Table IV.

Both samples (N-containing and reference) present similar values of activation energy for localized excitons, which are associated to a similar density of structural defects introduced during the growth at similar lower temperatures. However, differences in the activation energies and radiative lifetimes ratios for the delocalized excitons' processes reflect a slight different localization effect for the $\mathrm{N}$-containing sample which persists at higher temperatures.

We observed a higher intensity for the thinner QW in the nitrogenated sample as compared to the thicker QW. To ascribe this contrast to confinement effects, we can emulate the wave functions, $\Psi_{e(h h)}=F_{e(h h)}(z) \phi_{e(h h)}(r, \theta)$, within a finite barrier QW, $V_{e}(z)$ for electrons and $V_{h h}(z)$, for holes, according to the corresponding band offsets at the

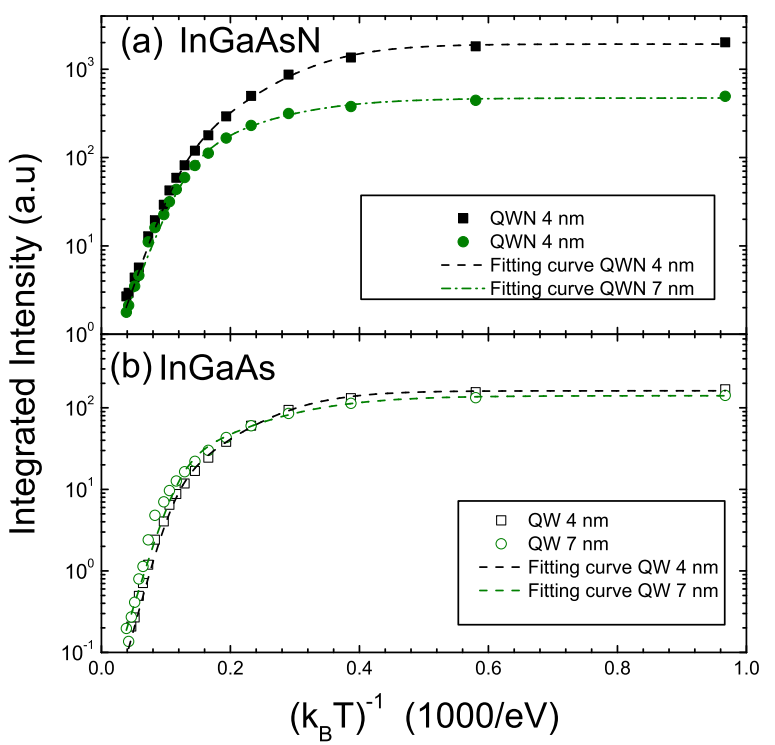

FIG. 6. Integrated PL intensity as function of the inverse of temperature for both samples. 
TABLE IV. Fitted parameters obtained by Arhenius plot.

\begin{tabular}{lccccc}
\hline \hline Parameters & $I_{0}(\mathrm{a} . \mathrm{u})$ & $\gamma(1)$ & $E_{a}(1) \mathrm{meV}$ & $\gamma(2)$ & $E_{a}(2) \mathrm{meV}$ \\
\hline QW 4 nm & 162 & 58 & 15 & 25000 & 68 \\
QW 7 nm & 141 & 17 & 11 & 7500 & 60 \\
QWN 4 nm & 1900 & 78 & 14 & 6400 & 50 \\
QWN 7 nm & 470 & 14 & 11 & 1500 & 46 \\
\hline \hline
\end{tabular}

conduction and valence bands and a tunable effective lateral cylindrical confinement with effective radius $R$ given by the Hamiltonian

$$
H_{e(h h)}=\frac{p^{2}}{2 m_{e(h h)}}+V_{e(h h)}(z)+\frac{\hbar^{2}}{2 m_{o} R^{4}} r^{2},
$$

with $p=-i \hbar \nabla+e / \mathrm{cA}, m_{o}$-the bare electron mass, and $A=B / 2(-y, x, 0)$, under magnetic field strength, $B$. In this approximation, the spin does not affect the wave function, thus its effect was not considered in the last equation. Then, the overlap integrals $\left|\left\langle F_{e}(z) \mid F_{h h}(z)\right\rangle\right|^{2}$ and $\left|\left\langle\phi_{e}(r, \theta) \mid \phi_{h h}(r, \theta)\right\rangle\right|^{2}$ can be calculated as given in Fig. 7 .

It can be clearly seen in Fig. 7(a) that by only reducing the QW width would not produce an increase of in the oscillator strength proportional to the overlap of wave functions. Yet, promoting a lateral effective confinement, either by reducing the value of the effective radius, $R$, or by increasing the magnetic field strength, as shown in Fig. 7(b), the probability of the electron-hole recombination can be enhanced.
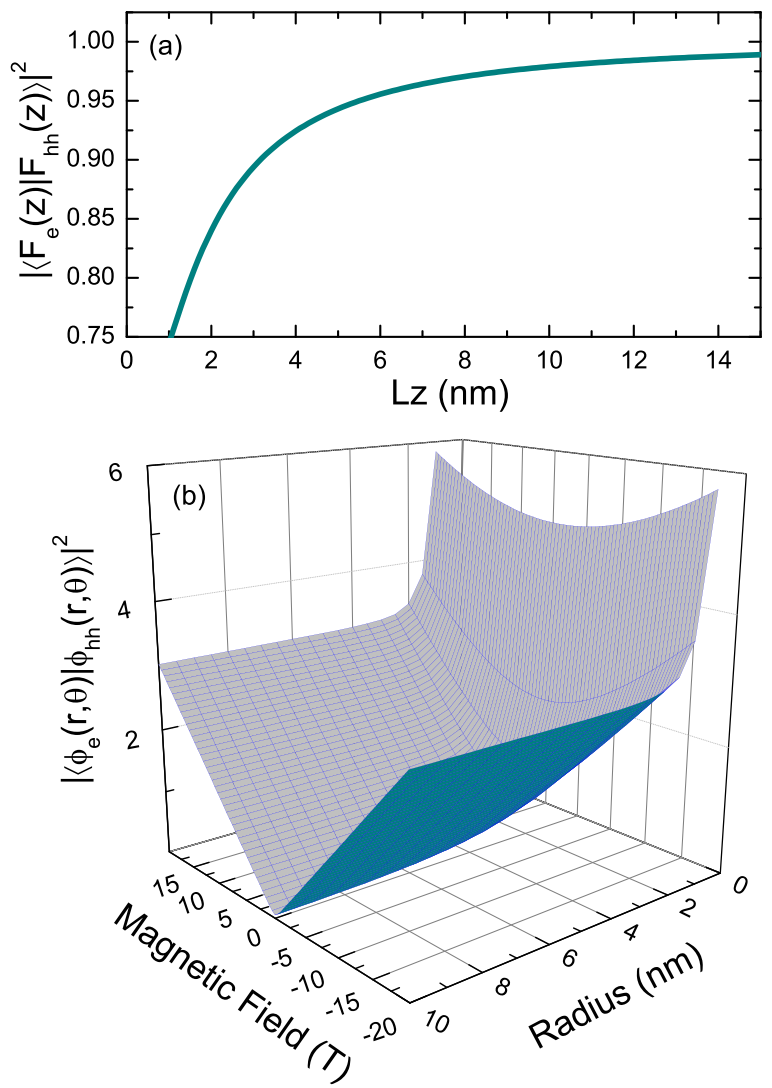

FIG. 7. (a) Calculated overlap integral for a GaInAsN/GaAs QW as a function of its width, $L z$. (b) Calculated overlap integral for the in-plane wavefunctions as a function of the lateral confinement radius, $R$, and magnetic field.
Figures 8(a) and 8(b) show the polarization-resolved PL spectra at a low temperature for $B=0 \mathrm{~T}$ and $B=15 \mathrm{~T}$. We have determined the diamagnetic shift and spin-splitting as well as the polarization degree of optical emission.

A reduction in the diamagnetic shift is observed for $\mathrm{N}$-containing samples, which indicates an increase in the electron-hole pair reduced mass as discussed below. We also observed an anomalous behaviour for the magnetic shift of the $4 \mathrm{~nm}$ InGaAsN QW. It should be highlighted that this effect can be well ascribed to both strain and confinement modulation. The non-linear behavior of the magnetic shift of the valence band ground state calculated from the multiband k.p Hamiltonian was characterized in Fig. 9(a) for positive strain in an InGaAs QW. Yet, even for negative strains, as reported experimentally, a decreasing aspect ratio in confined hole states at the interfaces may lead to a drastic nonlinear response as shown for a InGaAsN, $4 \mathrm{~nm}$, QW in Fig. 9(b). Note that negative values of $m_{x y}^{h}(B)$ can be attained at low fields. This will ultimately lead to increases in the reduced mass $\mu_{x y}=m_{x y}^{e} /\left(1-m_{x y}^{e} /\left|m_{x y}^{h}\right|\right)$, flattening the magnetic shift of the electron-hole pair emission peak, as observed in the experiment in Fig. 8 or even an inversion of its sign, turning the electron-hole magnetic shift negative. This effect has already been predicted and measured for confined excitons. ${ }^{27,28}$

For a qualitative discussion, let us assume the magnetic energy of a trapped electron as given by an effective Hamiltonian and presented in Eq. (17). In this case, the solution for the ground state energy due to the lateral and
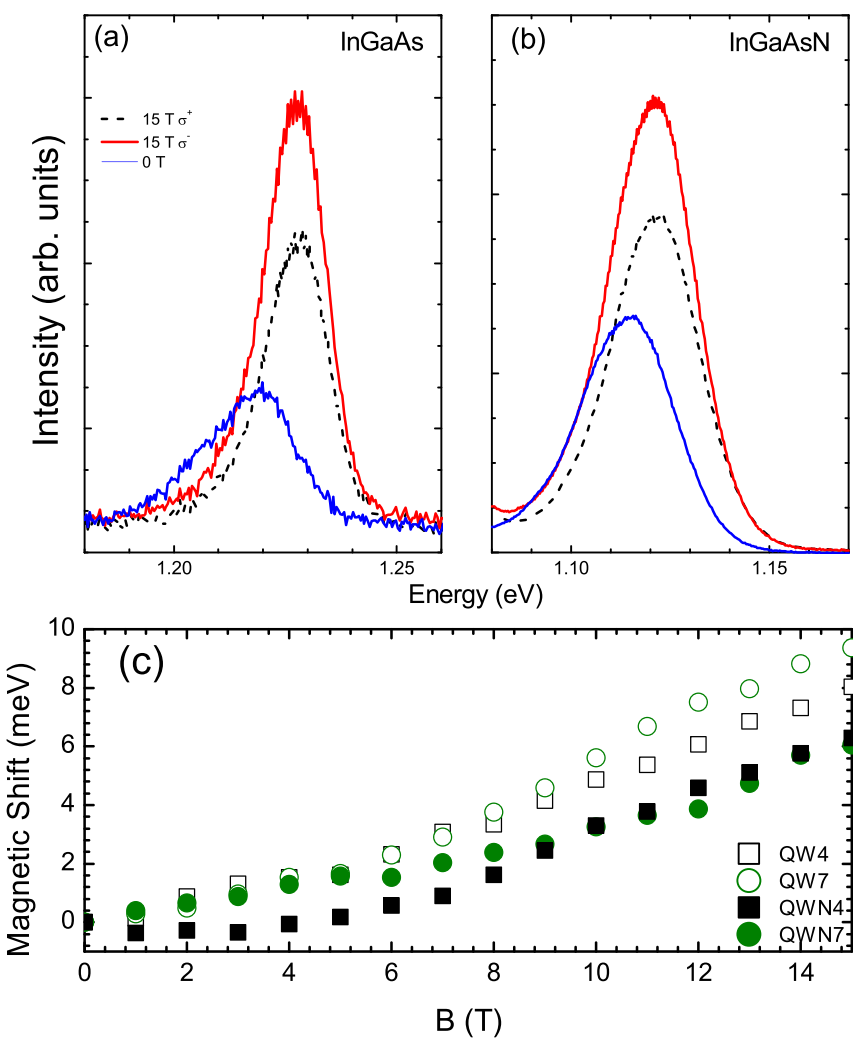

FIG. 8. Polarized resolved PL spectra under 0 and $15 \mathrm{~T}$ at $6 \mathrm{~K}$ for QW4 (a) InGaAs and (b) InGaAsN (c) magnetic shift as function of magnetic field for both InGaAs and InGaAsN QWs. 

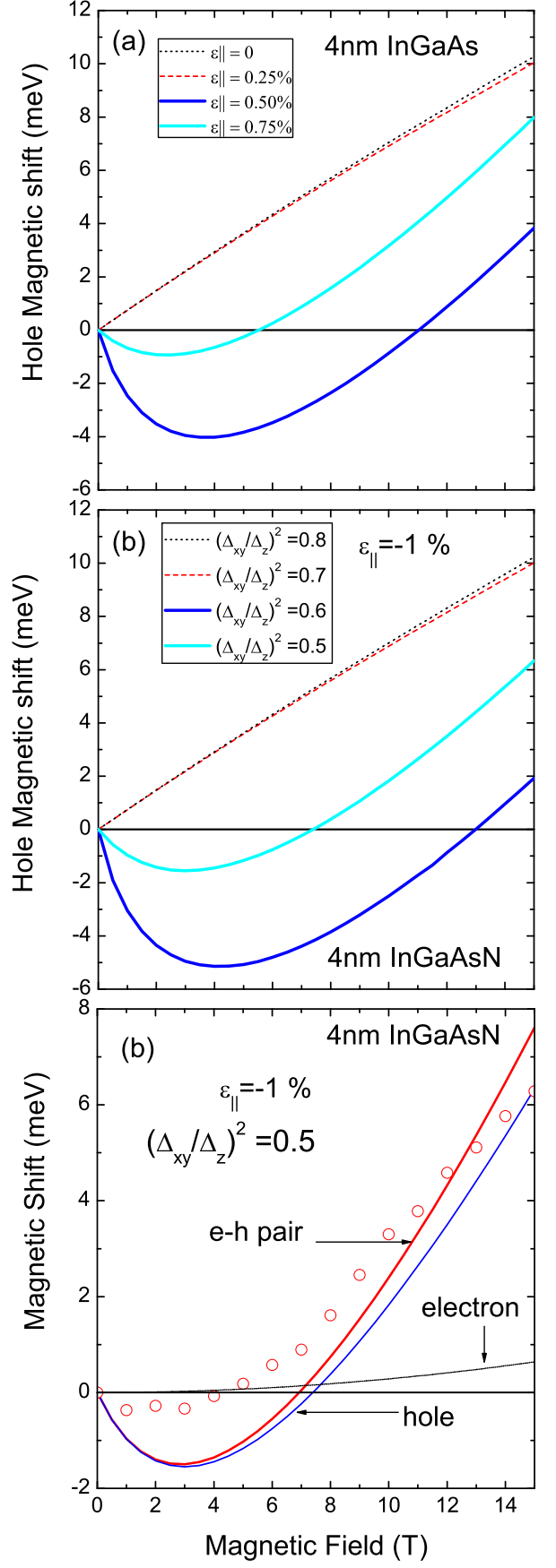

FIG. 9. Calculated magnetic shift for: (a) the holes at the ground state of the $4 \mathrm{~nm} \mathrm{InGaAs} \mathrm{QW} \mathrm{for} \mathrm{various} \mathrm{values} \mathrm{of} \mathrm{the} \mathrm{in-plane} \mathrm{strain,} \mathrm{(b)} \mathrm{the} \mathrm{holes}$ ground state in a $4 \mathrm{~nm} \mathrm{InGaAsN} \mathrm{QW} \mathrm{at} \mathrm{a} \mathrm{fixed} \varepsilon_{\|}=-1 \%$ for various values of the square aspect ratio. (c) Calculated magnetic shift in a $4 \mathrm{~nm} \mathrm{InGaAsN}$ QW for electrons, holes and electron-hole pair. The symbols represent the experimental results.

magnetic confinement (the mean value over the spin-split states) is given by

$$
E_{e}^{x y}=\frac{\hbar^{2}}{\sqrt{m_{x y}^{*}} m_{o} R^{2}}\left[1+\frac{1}{4 m_{x y}^{*}}\left(\frac{e B R^{2}}{c \hbar}\right)^{2}\right]^{\frac{1}{2}} .
$$

By using the electron mass reported in Table I, we calculated the effective magnetic shift for the single particle energy according to Eq. (18) and plotted it in Fig. 9(c) for an
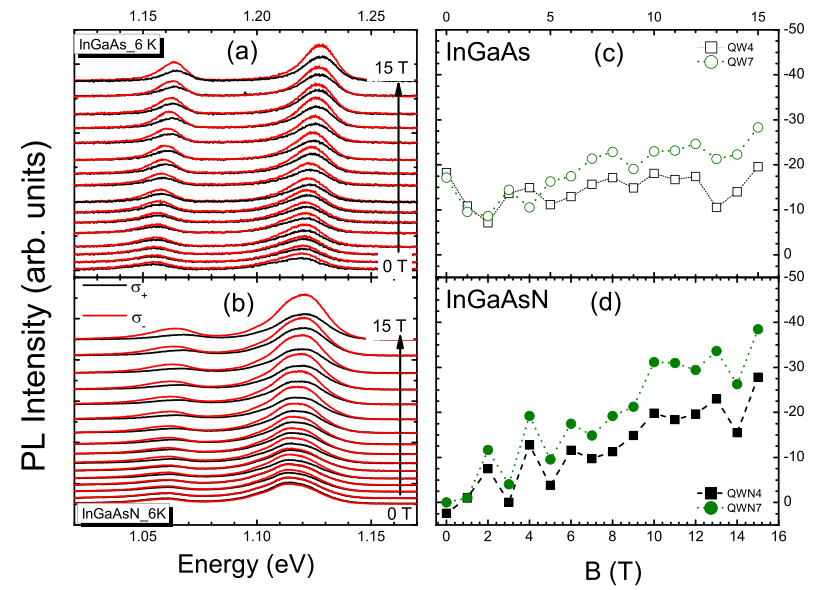

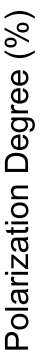

FIG. 10. Polarized resolved PL spectra for magnetic fields up to $15 \mathrm{~T}$ for (a) InGaAs and (b) InGaAsN quantum wells. (c) Circularly optical polarization as function of magnetic field for both (c) InGaAs and (d) InGaAsN QWs.

effective radius of the lateral confinement $R=1.2 \mathrm{~nm}$. This result would correspond to the weak magnetic field regime discussed in Ref. 6 when

$$
B<\frac{2 \sqrt{m_{x y}^{*}} \hbar c}{e R^{2}} .
$$

Then, by adding the electron shift to the hole magnetic shift one obtains the net relative electron-hole energy modulation is obtained, which is also shown. Thus, the non-linear magnetic shift observed experimentally can be ascribed to the field tuning of the hole effective mass, $m_{x y}^{h}(B)$, due to nonparabolicity and inter-subband coupling.

Additional features of the spin response under a magnetic field were revealed with circular optical polarization. Figures 10(a) and 10(b) show the polarized resolved spectra for InGaAs and InGaAsN QW, respectively, in the $0-15 \mathrm{~T}$ range. The polarization degree was calculated as follows:

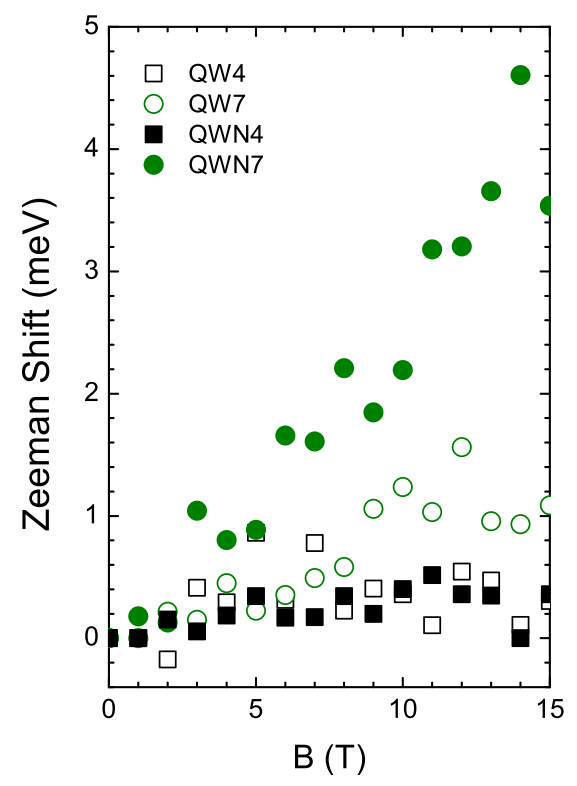

FIG. 11. Zeeman splitting for InGaAs (blank symbols) and InGaAsN (filled symbols) QW as a function of magnetic field. 


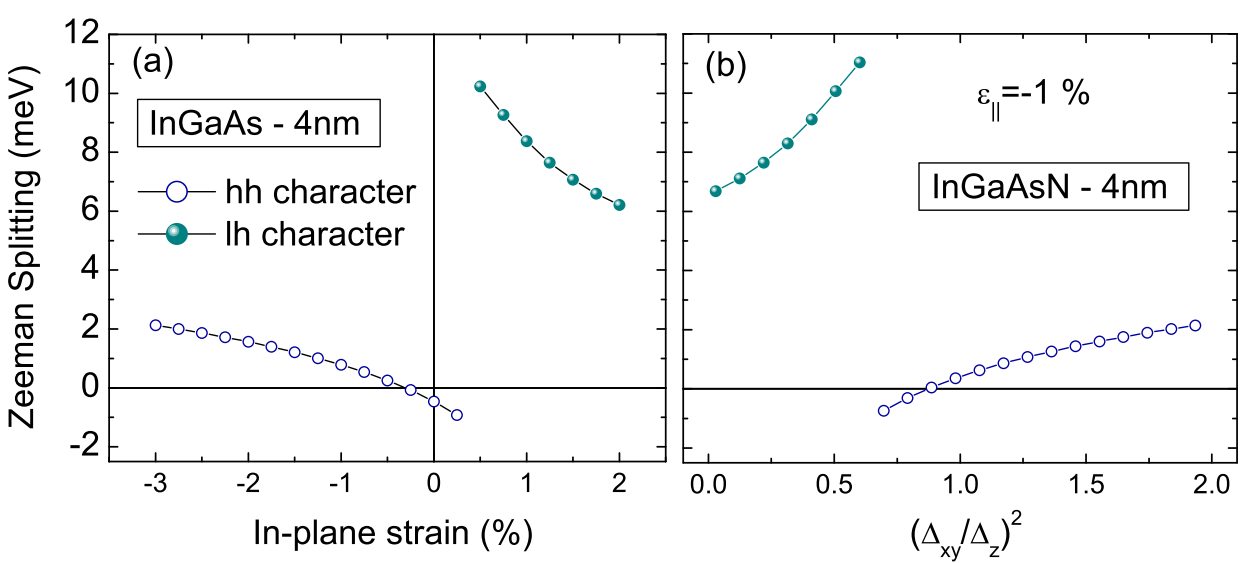

where $I(\sigma)$ is the intensity of the PL signal with respect to the polarizations $\sigma^{-}$and $\sigma^{+}$.

We note two important aspects: (i) a finite polarization degree was detected at $0 \mathrm{~T}$ for the InGaAs QW and (ii) there are unambiguous polarization fluctuations as the magnetic field increases in the InGaAsN QW. The first phenomenon has been reported for some systems ${ }^{36}$ and it is under investigation, while the second one can be attributed to the spin dynamics during carrier relaxation at the conduction band. ${ }^{37}$ We have observed that the polarization degrees for the $\mathrm{N}$-2 containing samples are slightly higher than for $\mathrm{N}$-free samples and the spin-dependent effects are stronger for wider QWs for both systems. Remark that under higher magnetic fields, the spin polarization degree remains within the range of $[-20,-10 \%]$, regardless of the structural parameters of the QW. Yet, for the spin-splitting, as shown in Fig. 11, a more significant dependence on the QW composition and size was attained.

It can be observed that there was an increase in excitonic spin-splitting for N-containing QWs. Particularly, at higher fields, the spin-splitting is around $1.3 \mathrm{meV}$ for the reference QW7, while for N-containing QW it is around $4.0 \mathrm{meV}$. However, the polarization degree, although affected directly by the increase in the excitonic spin-splitting, does not follow the same trends.

For the characterization of the magneto-optical response and its correlation with the electronic structure, we shall focus on relevant valence band effects. The magnetic field effects in the valence band are presented in Fig. 12, where the Valence Band Zeeman Splitting of the ground state at $15 \mathrm{~T}$ is shown. It can observed that the character change around $0.3 \%$, also presented in Fig. 1 leads to a drastic shift in this magnetic splitting. It is also important to highlight the sign inversion of the splitting by varying the values of the compressive strain, where the character of the ground state is predominantly $h h$.

An equivalent behavior would be expected by decreasing the aspect ratio, $\left(\frac{\Delta_{x y}}{\Delta_{z}}\right)^{2}$, as indicated in the upper axes. Thus, the effect of the compressive strain is counterbalanced by a decrease of the aspect ratio in a confined state at the interfaces. The aspect ratio decrease can even tune a character change of the valence band ground state making it more lh-like. The values obtained for the valence band spin splitting in the vast part of the parameter range, shown in Fig. 12 (calculated at $B=15 \mathrm{~T}$ ), are in accordance with the values detected in the experiments for the electron-hole pair recombination. Yet, close to the parameter boundary where a character change takes place at the ground state, sudden changes of the magnetic response can be expected.

According to the values represented in Fig. 12, we calculated the degree of spin polarization (DSP) of the valence band ground state, shown in Fig. 13. We used the equation ${ }^{38}$

$$
D S P=\frac{\Delta E_{\uparrow \downarrow}}{\left|\Delta E_{\uparrow \downarrow}\right|}\left[\frac{\exp \left(\frac{-\left|\Delta E_{\uparrow \downarrow}\right|}{k_{B} T}\right)-1}{\left(1+\frac{\tau_{s}}{\tau}\right)+\exp \left(\frac{-\left|\Delta E_{\uparrow \downarrow}\right|}{k_{B} T}\right)}\right],
$$
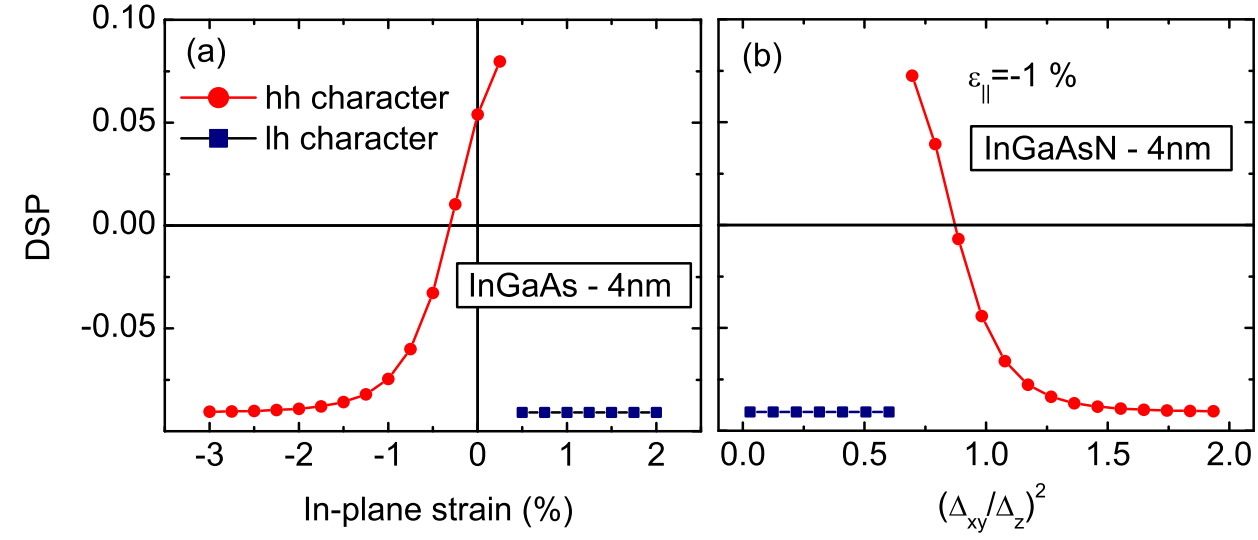

FIG. 13. Calculated degree of spin polarization for the valence band ground state at $15 \mathrm{~T}$, for: (a) the $4 \mathrm{~nm}$ $\mathrm{In}_{0.36} \mathrm{Ga}_{0.64}$ As quantum well at $\mathrm{T}=2 \mathrm{~K}$ as a function of the in-plane strain and (b) for the $4 \mathrm{~nm} \operatorname{In}_{0.36} \mathrm{Ga}_{0.64} \mathrm{As}_{0.088}$ $N_{0.012}$ QW at a fixed $\varepsilon_{\|}=-1 \%$ as a function of the square aspect ratio. 
where $\Delta E_{\uparrow \downarrow}$ is the energy difference between the spin-up and down states. The ratio $\tau_{s} / \tau$ was set to 0.1 that is reasonable for QWs of this size. ${ }^{38}$ Note in this case that despite the monotonic variation of the Zeeman splitting described before as a function of the structural parameters (strain and aspect ratio of the confined state), the DSP for holes in Fig. 13 follows very flat behaviors at $T=2 \mathrm{~K}$ below and above the critical parameter region $\left(\varepsilon_{\|} \sim 0.5\right.$ in Fig. 13 (a) or $\Delta_{x y} / \Delta_{x y} \sim 0.5$ Fig. 13(b)), where the character of the valence ground state changes. The valence band DSP is, under these conditions, close to $-10 \%$, at $B=15 \mathrm{~T}$. Thus, the experimental observation of a slight dependence of the polarization degree on the structural parameters can, in principle, be ascribed to the spin polarization of the valence band ground state. Therefore, systems with an n-type character should have the polarization defined by the minority carriers at the valence band.

\section{CONCLUSIONS}

The experimental and theoretical magnetic response of electron-hole pairs in InGaAsN quantum wells were investigated and correlated. Our results reveal peculiar behavior for hole spin polarization and diamagnetic shift as function of magnetic field, strain modulation, and carrier localization. It was shown that the spin polarization degree is dependent of the character of valence band ground state. The observed anomalous behavior of the electron-hole pair magnetic shift at low magnetic fields was associated to the increase in the exciton reduced mass due to the negative effective mass of the valence band ground state which results in the observation of negative magnetic shifts. Finally, our results show that the valence band effects are an important issue for the physical properties of dilute nitrite semiconductor nanostructures.

\section{ACKNOWLEDGMENTS}

The authors are grateful to Professor Maria Jose S.P. Brasil from UNICAMP (Universidade Estadual de Campinas-Brazil) for the magneto-PL measurements and the Brazilian Agencies FAPESP Procs: 2013/17657-2, 2012/ 24055-6, 2012/02655-1, and 2011/17944-6 Capes grant: A067-2013, as well as CNPq for the financial support.

${ }^{1}$ W. Shan, W. Walukiewicz, J. W. Ager III, E. E. Haller, J. F. Geisz, D. J. Friedman, J. M. Olson, and S. R. Krutz, Phys. Rev. Lett. 82, 1221 (1999).

${ }^{2}$ L. Grenouillet, C. Bru-Chevallier, G. Guillot, P. Gilet, P. Duvaut, C. Vannuffel, A. Million, and A. Chenevas-Paule, Appl. Phys. Lett. 76, 2241 (2000).

${ }^{3}$ M.-A. Pinault and E. Tourni, Appl. Phys. Lett. 78, 1562 (2001).

${ }^{4}$ Z. Pan, L. H. Li, Y. W. Lin, B. Q. Sun, D. S. Jiang, and W. K. Ge, Appl. Phys. Lett. 78, 2217 (2001).
${ }^{5}$ S. Mazzucato, A. Erol, A. Teke, M. C. Arikan, R. J. Potter, N. Balkan, X. Marie, A. Boland-Thoms, H. Carrere, E. Bedel, G. Lacoste, C. Fontaine, and A. Arnoult, Physica E 17, 250 (2003).

${ }^{6}$ T. Nuytten, M. Hayne, B. Bansal, H. Y. Liu, M. Hopkinson, and V. V. Moshchalkov, Phys. Rev. B 84, 045302 (2011).

${ }^{7}$ H. Saito, T. Makimoto, and N. Kobayashi, J. Cryst. Growth 170, 372 (1997).

${ }^{8}$ M. Latkowska, R. Kudrawiec, G. Sek, J. Misiewicz, J. Ibanez, M. Hopkinson, and M. Henini, Phys. Status Solidi C 8, 1655 (2011); Appl. Phys. Lett. 98, 131903 (2011).

${ }^{9}$ A. Polimeni, M. Capizzi, M. Geddo, M. Fischer, M. Reinhardt, and A. Forchel, Phys. Rev. B 63, 195320 (2001).

${ }^{10}$ A. Polimeni, F. Masia, A. Vinattieri, G. Baldassarri Hger von Hgersthal, and M. Capizzi, Appl. Phys. Lett. 84, 2295 (2004).

${ }^{11}$ H. Carrre, A. Arnoult, A. Ricard, and E. Bedel-Pereira, J. Cryst. Growth 243, 295 (2002).

${ }^{12}$ T. Kageyama, T. Miyamoto, S. Makino, F. Koyama, and K. Iga, Jpn. J. Appl. Phys. 38, L298 (1999).

${ }^{13}$ S. Tomić and E. P. O'Reilly, Phys. Rev. B 71, 233301 (2005).

${ }^{14}$ A. Lindsay and E. P. O'Reilly, Phys. Rev. Lett. 93, 196402 (2004).

${ }^{15}$ G. E. Pikus and G. L. Bir, Sov. Phys. Solid. State 1, 136 (1959).

${ }^{16}$ A. Khatab, M. Shafi, R. H. Mari, M. Aziz, M. Henini, G. Patriarche, D. Troadec, M. Sadeghi, and S. Wang, Phys. Status Solidi C 9, 1621 (2012).

${ }^{17}$ J. M. Luttinger, Phys. Rev. 102, 1030 (1956).

${ }^{18}$ G. L. Bir and G. E. Pikus, Symmetry and Strain-Induced Effects in Semiconductors (Wiley, New York, 1974), p. 311.

${ }^{19}$ R. Kudrawiec, M. Motyka, M. Gladysiewicz, J. Misiewicz, J. A. Gupta, and G. C. Aers, Solid State Commun. 138, 365-370 (2006).

${ }^{20}$ O. Rubel, M. Galluppi, S. D. Baranovskii, K. Volz, L. Geelhaar, H. Riechert, P. Thomas, and W. Stolz, J. Appl. Phys. 98, 063518 (2005).

${ }^{21}$ R. Kudrawiec, M. Latkowska, M. Baranowski, J. Misiewicz, L. H. Li, and J. C. Harmand, Phys. Rev. B 88, 125201 (2013).

${ }^{22}$ S. Adachi, J. Appl. Phys. 53, 8775 (1982).

${ }^{23}$ Yu. F. Biryulin, N. V. Ganina, M. G. Mil'vidskii, V. V. Chaldyshev, and Yu. V. Shmartsev, Sov. Phys. Semicond. (English Transl.) 17, 68 (1983).

${ }^{24}$ I. Vurgaftman and J. R. Meyer, J. Appl. Phys. 94, 3675 (2003).

${ }^{25}$ J. Ibañez, R. Kudrawiec, J. Misiewicz, M. Schmidbauer, M. Henini, and M. Hopkinson, J. Appl. Phys. 100, 093522 (2006).

${ }^{26}$ L. Xu, D. Patel, C. S. Menoni, J. Y. Yeh, L. J. Mawst, and N. Tansu, Appl. Phys. Lett. 89, 171112 (2006).

${ }^{27}$ E. Margapoti, Fabrizio M. Alves, S. Mahapatra, T. Schmidt, V. LopezRichard, C. Destefani, E. Menendez-Proupin, F. Qu, C. Bougerol, K. Brunner, A. Forchel, G. E. Marques, and L. Worschech, Phys. Rev. B 82, 205318 (2010).

${ }^{28}$ E. Margapoti, Fabrizio M. Alves, S. Mahapatra, T. Schmidt, V. LopezRichard, C. Destefani, E. Menendez-Proupin, F. Qu, C. Bougerol, K. Brunner, A. Forchel, G. E. Marques, and L. Worschech, New. J. Phys. 14, 043048 (2012).

${ }^{29}$ D. Schlenker et al., J. Cryst. Growth 196, 67 (1999).

${ }^{30}$ W. Li, M. Pessa, and J. Likonen, Appl. Phys. Lett. 78, 2864 (2001).

${ }^{31}$ Y. P. Varshni, Physica 34, 149 (1967).

${ }^{32}$ I. Vurgaftman, J. R. Meyer, and L. R. Ram-Mohan, J. Appl. Phys. 89, 5815 (2001).

${ }^{33}$ A. Polimeni, M. Capizzi, M. Geddo, M. Fischer, M. Reinhardt, and A. Forchel, Appl. Phys. Lett. 77, 2870 (2000).

${ }^{34}$ L. C. Andreani, Solid State Comm. 77, 641 (1991).

${ }^{35}$ D. S. Citrin, Phys. Rev. B 47, 3832 (1993).

${ }^{36}$ X. J. Wang, I. A. Buyanova, F. Zhao, D. Lagarde, A. Balocchi, X. Marie, C. W. Tu, J. C. Harmand, and W. M. Chen, Nature Mater. 8, 198 (2009).

${ }^{37}$ L. Fernandes dos Santos, Yu. A. Pusep, L. Villegas-Lelovsky, V. LopezRichard, G. E. Marques, G. M. Gusev, D. Smirnov, and A. K. Bakarov, Phys. Rev. B 86, 125415 (2012).

${ }^{38}$ L. K. Castelano, D. F. Cesar, V. Lopez-Richard, G. E. Marques, O. D. D. Couto, Jr., F. Iikawa, R. Hey, and P. V. Santos, Phys. Rev. B 84, 205332 (2011). 